\title{
PREEMPTIVE GASTROINTESTINAL TRACT MANAGEMENT REDUCES ASPIRATION AND RESPIRATORY FAILURE AFTER THORACIC OPERATIONS
}

John R. Roberts, MD

Yu Shyr, PhD

Karla R. Christian, MD

Davis Drinkwater, MD

Walter Merrill, MD
Objectives: Respiratory failure is the major mode of death after general thoracic operations. However, respiratory failure may develop from two very different mechanisms: aspiration, often caused by ileus, and pneumonia, which often results from poor pain control. Epidural catheters help control pain and prevent pneumonia but contribute to ileus and may increase aspiration. We report a decrease in the incidence of aspiration after changing postoperative care to include gastrointestinal tract management. Methods: All patients undergoing elective thoracotomy by a single surgeon were evaluated for hospital mortality and morbidity. For the first 21 months, patients did not receive an intraoperative nasogastric tube and were prescribed an "advance as tolerated" diet after the operation $(n=125)$. For the second period, nasogastric tubes were placed intraoperatively and patients received nothing by mouth the day of operation, clear liquids the first day, and a regular diet the second day ( $n=153$ ). Pneumonia was considered to have developed if infiltrates developed in a single lobe or two adjoining lobes and culture of the sputa grew a dominant organism. Patients were considered to have aspirated if diffuse infiltrates developed or cultures grew multiple organisms. Significance of results was determined by $\chi^{2}$ testing. Results: A total of 278 patients underwent elective lung resection over a $3 \frac{1}{2}-$ year period, 125 with ad libitum dietary management and 153 with intensive management of the gastrointestinal tract. Six patients $(4.84 \%)$ aspirated before the institution of gastrointestinal tract management, whereas none $(\mathbf{0 . 0} \%)$ aspirated after the change. This difference was significant $(P=.01)$. Respiratory mortality was eliminated in the group with gastrointestinal tract management $(P=.04)$. Conclusions: Aspiration and its subsequent respiratory failure and mortality can be decreased with preemptive gastrointestinal tract management. (J Thorac Cardiovasc Surg 2000;119:449-52)
$\mathrm{M}$ any studies on perioperative mortality after lung resection and thoracotomy have concluded that respiratory failure is the most common cause of death. ${ }^{1,2}$ However, respiratory failure can result from

From the Department of Cardiac and Thoracic Surgery, Vanderbilt University, Nashville, Tenn.

Read at the Seventy-ninth Annual Meeting of The American Association for Thoracic Surgery, New Orleans, La, April 18-21, 1999.

Received for publication April 22, 1999; revisions requested June 28, 1999; revisions received Oct 25, 1999; accepted for publication Oct 26, 1999

Address for reprints: John R. Roberts, MD, Department of Cardiac and Thoracic Surgery, Vanderbilt University, 2986 TVC, Nashville, TN 37232 (E-mail: bob.roberts@mcmail.vanderbilt.edu). Copyright @ 2000 by Mosby, Inc.

0022-5223/2000 $\$ 12.00+0 \quad \mathbf{1 2 / 6 / 1 0 4 0 3 0}$

doi: $10.1067 / \mathrm{mtc} .2000 .104030$ many different causes, and it can be a primary cause of illness (as in pneumonia or aspiration) or a secondary failure as a general response to major stress (congestive heart failure, sepsis, or hypotension).

Pneumonia and aspiration are the two most dangerous causes of primary respiratory failure in patients undergoing lung resection. We believe that pneumonia develops in patients with poor pain control and weak cough, whereas aspiration occurs in patients with impaired or delayed gastrointestinal tract transit. Epidural catheters clearly help treat pain but may contribute to ileus. Further, we believe that recovery from aspiration after lung resection is a long, tedious process and less generally successful than recovery from pneumonia.

Either primary or secondary failure can lead to adult respiratory distress syndrome (ARDS). Although the 
Table I. Patient demographics

\begin{tabular}{lccc}
\hline & Before 7/1/97 & After 7/1/97 & P value \\
\hline No. of patients & 124 & 151 & \\
Men & 80 & 97 & .96 \\
Women & 44 & 54 & \\
Age (y) & $59 \pm 14$ & $60 \pm 15$ & .86 \\
Lobectomies & 49 & 81 & \\
Complicated lobectomies & 21 & 26 & \\
Pneumonectomies & 18 & 17 & \\
Decortications & 36 & 27 &
\end{tabular}

Analysis of patient demographics revealed no significant difference between the two procedures in fraction of men or in type of procedure.

survival after the development of ARDS has been reported to be as low as $50 \%$, anecdotal reports suggest that the survival after the development of ARDS in the setting of lung resection is less. Thus prevention of the complication is paramount.

In response to two episodes of aspiration, the primary surgeon in this study (J.R.R.) changed his management policy from an ad libitum dietary advancement to one of routine gastric drainage perioperatively and dietary control in hopes of decreasing the incidence of aspiration.

\section{Methods}

All patients undergoing elective thoracotomy by a single surgeon at several hospitals over 42 months were included in the study. The study was limited to a single surgeon's experience because the change in management was not consistent with the management done by the other surgeons in the same practice. All patients undergoing thoracotomy and resections requiring lobectomy, complicated lobectomy (including chest wall resections, diaphragm resections, sleeve resections of artery or bronchus, and lobectomies in patients with a forced expiratory volume in 1 second $<1 \mathrm{~L}$ ), decortication, or pneumonectomy were given epidural anesthesia and chest physiotherapy and early ambulation. Patients were initially given clear liquid diets and diet was advanced as tolerated after the operation. Cathartics were given as necessary to promote bowel activity. The remainder of gastrointestinal tract management was directed toward identifying ileus and treating it when clinically manifest.

After two episodes of apparent aspiration in 1 week, gastrointestinal tract management was altered to diminish the chance of aspiration. Nasogastric tubes were placed intraoperatively and removed in the recovery room if less than 300 $\mathrm{mL}$ of liquid was obtained during the operation. Dietary management was nothing by mouth the day of the operation, clear liquids the first postoperative day, and regular diets the second postoperative day. Nasogastric tubes were replaced after any episode of vomiting, and performance of daily abdominal examinations to identify ileus was stressed to the residency
Table II. Distribution of procedures

\begin{tabular}{lccc}
\hline & Before 7/1/97 & After 7/1/97 & P value \\
\hline Lobectomies & $49(39.5 \%)$ & $81(53.6 \%)$ & .02 \\
Pneumonectomies & $18(14.5 \%)$ & $17(11.3 \%)$ & .47 \\
Neoadjuvant & $13(10.5 \%)$ & $24(15.9 \%)$ & .03 \\
Decortication & $36(29.0 \%)$ & $27(17.9 \%)$ & .03 \\
Complicated lobectomies & $21(17.0 \%)$ & $26(17.4 \%)$ & .99 \\
\hline
\end{tabular}

and nursing staff. Fifty-four patients (36\%) had nasogastric tubes in place overnight. Fourteen patients $(9 \%)$ required replacement of nasogastric tubes because of abdominal distention or vomiting. If ileus was identified, a nothing by mouth order was given and patients were followed up.

Data analyzed included age, sex, pulmonary function test results, types of procedures, mortality, respiratory mortality, morbidity, life-threatening complications, tracheostomy, and reintubation. Patients were considered to have aspirated if diffuse infiltrates developed or culture of the sputa grew multiple organisms. Patients were considered to have pneumonia if they had infiltrates in one or two adjoining lobes and culture of the sputa grew a single dominant organism. Mortality was defined as hospital mortality, regardless of whether this occurred before or after 30 days. Life-threatening complications were defined as those that prompted intubation, cardioversion, or emergency operation or caused sepsis. Patients underwent a variety of procedures, including lobectomies, pneumonectomies, decortications, and complicated lobectomies.

The Student $t$ test was used to assess differences in means, and the Fisher exact test was used to assess differences in proportions. This was a comparison study of sequential groups of patients. Because the event being studied is an extremely low-frequency event, the usual adjustments for nonrandomized trials that attempt to adjust for selection factors were powerless in this low-frequency setting.

\section{Results}

A total of 275 patients underwent thoracotomy, 124 before and 151 after the change in gastrointestinal tract management. Table I lists the patients and their demographic analyses. No differences in proportion of men and women, age, or pulmonary function were found. Table II summarizes the proportions of procedures. Differences in proportions of lobectomies and of decortications were found, with relatively more lobectomies in the second group and relatively more decortications in the first.

Table III summarizes the mortality data. Four of 124 patients died before hospital discharge in the first group. All of these patients died of respiratory causes for an overall and respiratory mortality rate of $3.23 \%$. In the second group, 2 of 151 patients died (bowel 
Table III. Mortality

\begin{tabular}{lccc}
\hline & Before 7/1/97 & After 7/1/97 & P value \\
\hline Respiratory mortality & $4(3.23 \%)$ & $0(0.00 \%)$ & .04 \\
Mortality & $4(3.23 \%)$ & $2(1.32 \%)$ & .41 \\
\hline
\end{tabular}

Analysis of the effect on mortality suggested a decrease in respiratory mortality with the preemptive gastrointestinal tract management.

ischemia, empyema) for an overall mortality rate of $1.23 \%$. There were no respiratory deaths in the second group, so that the respiratory mortality rate was $0.00 \%$. The difference in overall mortality was not significant, whereas the difference in respiratory mortality was significant $(P=.04)$.

Respiratory morbidity is summarized in Table IV. The incidence of aspiration as defined in this study was entirely eliminated and represented a significant change. No significant changes in pneumonia and reintubation were found. Other factors were analyzed (incidence of tracheostomy and of life-threatening complications) but did not reach statistical significance.

\section{Discussion}

A surgeon provides two primary services to a patient with cancer: removing the tumor with disease-free margins and getting the patient safely through the procedure. To that end, analyses of care patterns that can improve the likelihood of safe operations get at the heart of what cancer surgeons do.

Kohman and colleagues ${ }^{3}$ analyzed 37 different factors that might affect the morbidity and mortality of lung resections and determined that preoperative factors only predict $28 \%$ of the risk in patients undergoing lung resection. They concluded that $72 \%$ of the complications must be attributed to factors not considered or to random factors. Our data indicate that issues of surgical management, specifically aspiration, might account for a portion of, or can decrease the impact of, these random factors.

Table V lists the largest series that report perioperative mortality after lobectomy and pneumonectomy. Mortality rates for lobectomy range from $0.47 \%$ in the Brigham series ${ }^{8}$ to as high as $4.03 \%$. The mortality rate for pneumonectomies ranges from $6.2 \%$ to $11.7 \%$. Nesbitt and associates ${ }^{10}$ recently reported an $8 \%$ perioperative mortality rate for all pneumonectomies done at the MD Anderson Cancer Center. These data all demonstrate that lung resection, whether by lobectomy or pneumonectomy, remains much more dangerous than a first-time cardiac bypass.

Whereas all these studies evaluated preoperative risk
Table IV. Respiratory morbidity

\begin{tabular}{lcrc}
\hline & Before $7 / 1 / 97$ & After 7/1/97 & P value \\
\hline Aspiration & $6(4.84 \%)$ & $0(0.00 \%)$ & .01 \\
Reintubation & $8(6.45 \%)$ & $12(7.95 \%)$ & .82 \\
Pneumonia & $3(2.42 \%)$ & $9(5.96 \%)$ & .24 \\
\hline
\end{tabular}

There was a significant decrease in aspiration with preemptive gastrointestinal tract management.

Table V. Perioperative mortality reported after lung resection

\begin{tabular}{|c|c|c|c|}
\hline Series & $\begin{array}{c}\text { Lobectomy } \\
(\%)\end{array}$ & $\begin{array}{c}\text { Pneumectomy } \\
(\%)\end{array}$ & Year \\
\hline LCSG (Ginsberg) ${ }^{1}$ & $2.9 *$ & $6.2 *$ & 1983 \\
\hline Keagy $^{4}$ & $2.2^{*}$ & & 1985 \\
\hline Kohman $^{3}$ & 3.7 & 11.7 & 1986 \\
\hline Roxburgh $^{5}$ & 2.7 & 6.7 & 1991 \\
\hline Romano $^{6}$ & 4.2 & 11.6 & 1992 \\
\hline Shah $^{7}$ & $4.03^{*}$ & $1.6^{*}$ & 1996 \\
\hline Roberts $^{8}$ & 0.47 & & 1996 \\
\hline Wada $^{9}$ & $1.2^{*}$ & $3.2^{*}$ & 1998 \\
\hline
\end{tabular}

An asterisk $(*)$ marks those series that reported 30-day mortality. The remaining series reported hospital mortality. LCSG, Lung Cancer Study Group.

factors in attempts to predict mortality, no good information identifies operative or perioperative management that might affect mortality. Further, no attempt has been made to separate the different causes of respiratory failure that might contribute to perioperative mortality.

Our data indicate that the chance of aspiration and the frequency of respiratory mortality can be decreased with gastrointestinal tract management that includes use of nasogastric tubes, dietary management, and frequent abdominal examinations. These are all techniques common to general surgical practice, and techniques that we apply routinely now to general thoracic surgical practice. Certainly many patients can be safely treated without such aggressive gastrointestinal tract management, as the mortality risk in our patients before our change indicates. However, the cost of a single aspiration, both in dollar terms and in mortality terms, is so high that significant measures to prevent it are justified.

We thank Richard R. Pierson, MD, for comments about the manuscript and Debbie Poteet for manuscript preparation.

\section{REFERENCES}

1. Ginsberg RJ, Hill LD, Eagan RT, Thomas P, Mountain CF, Deslauriers J, et al. Modern thirty-day operative mortality for surgical resections in lung cancer. J Thorac Cardiovasc Surg 1983; 86:654-8.

2. Naunheim KS, Kesler KA, D'Orazio SA, Fiore AC, Judd DR. 
Lung cancer surgery in the octogenarian. Eur J Cardiothorac Surg 1994;8:453-6.

3. Kohman LJ, Meyer JA, Ikins PM, Oates RP. Random versus predictable risks of mortality after thoracotomy for lung cancer. J Thorac Cardiovasc Surg 1986;91:551-4.

4. Keagy BA, Lores ME, Starek PJK, Murray GF, Lucas CL, Wilcox BR. Elective pulmonary lobectomy: factors associated with morbidity and operative mortality. Ann Thorac Surg 1985; 40:349-52.

5. Roxburgh JC, Thompson J, Goldstraw P. Hospital mortality and long-term survival after pulmonary resection in the elderly. Ann Thorac Surg 1991;51:800-3.

6. Romano PS, Mark DH. Patient and hospital characteristics related to in-hospital mortality after lung cancer resection. Chest 1992;101:1332-7.

7. Shah R, Sabanathan S, Richardson J, Mearns AJ, Goulden C. Results of surgical treatment of stage I and II lung cancer. J Cardiovasc Surg 1996;37:169-72.

8. Roberts JR, DeCamp MM, Mentzer SJ, Sugarbaker DJ. Prospective comparison of open and video-assisted lobectomy [abstract]. Chest 1996;110:45S.

9. Wada H, Nakamura T, Nakamoto K, Maeda M, Watanabe Y. Thirty-day operative mortality for thoracotomy in lung cancer. J Thorac Cardiovasc Surg 1998;115:70-3.

10. Nesbitt JC, Whitehead WG, Danush AA, et al. Survival and operative risk following pneumonectomy [abstract]. Chest 1997;112: $38 \mathrm{~S}$.

\section{Discussion}

Dr Scott J. Swanson (Boston, Mass). Do you see any downside to using the nasogastric tube during the first couple of days, and could that be contributing to why you might be seeing more cases of pneumonia?

Dr Roberts. I am not entirely sure now what the best timing is for the use of the nasogastric tube. Currently I put it in in the operating room and then take it out the night of the operation if the drainage has not been greater than $300 \mathrm{~mL}$. As you know, a nasogastric tube will decrease the patient's ability to cough, and it is certainly possible that the tube is contributing to pneumonia.

Dr Swanson. What size nasogastric tube were you using? Was it variable?

Dr Roberts. Variable, yes.

Dr Swanson. Do you think you would get the same benefit with a small tube but also potentially allow better clearance of secretions, or do you think function of the nasogastric tube would be a problem if it was too small?

Dr Roberts. That is a good suggestion. In some sense I think of the tube as a diagnostic maneuver rather than a therapeutic maneuver, at least initially, to find out if there are a lot of gastric contents. It may very well be that a small tube would be as useful as inserting the tube in the operating room and taking it out if there is not much drainage.

Dr Valerie W. Rusch (New York, NY). Can you tell us something about the type of epidural analgesia used? Were only narcotics used? Do your anesthesiologists use narcotics plus a local anesthetic? Is there a uniform policy in that regard?

Dr Roberts. We use a thoracic epidural that is a combina- tion of narcotics and local anesthetic. We typically vary it a little bit after it is in place if we have trouble with hypotension and decrease the amount of local anesthetic. By and large, however, it is pretty standard.

Dr Rusch. That may have some impact on the severity or duration of the ileus. Also, how many patients were actively smoking when they were admitted for the operation?

Dr Roberts. I do not have any data on that.

Dr Rusch. That really could be a confounding factor with respect to the frequency of postoperative respiratory problems.

Dr Roberts. Yes, I think it especially has an impact concerning pneumonia. Whereas I do not do volume-reduction operations in patients who are actively smoking, I do do lung cancer operations in patients who are still smoking; however, I do not have data on that.

Dr Mark K. Ferguson (Chicago, Ill). You are postulating that the epidural anesthetic contributes to the ileus. Are you leaving the epidural in until the chest tubes are removed?

Dr Roberts. Variably. Typically we leave the epidural in about 4 to 5 days if there is still an air leak, and then we usually take it out.

Dr Ferguson. I am curious as to how you think the nasogastric tube contributes if it is only left in overnight.

Dr Roberts. Overnight is the standard amount of time. If there is greater than $300 \mathrm{~mL}$ of fluid, then it is left in place. Several patients had them in for 4 or 5 days before the diet was advanced. In some sense, as I said, this is a diagnostic maneuver to determine whether the patients are having ileus in response to the operation and the epidural. If there is a lot of drainage at the end of the first day, then the tube stays in.

Dr Wickii T. Vigneswaran (Chicago, Ill). Can you comment about fluid management perioperatively? Did you change your management in any way? Did you actively use a vasoconstrictor because of the use of the epidural, which may have contributed to the bowel ischemia in one of the patients?

Dr Roberts. The one episode of bowel ischemia occurred on postoperative day 5 in an 80-year-old patient we were getting ready to send home, and I think his epidural had been out for a couple of days. I do not give patients any unneeded fluid. We do use dopamine and low-dose phenylephrine hydrochloride (Neo-Synephrine) for the immediate perioperative period, but the phenylephrine hydrochloride is always discontinued before they leave the recovery room later that day.

Dr Ferguson. Given some of the issues that have been raised and the potential importance of this, do you think a randomized trial is indicated, or are you sufficiently convinced?

Dr Roberts. At this point I am pretty convinced of the importance of it, and I have not considered whether a randomized trial would be appropriate. I have considered, from the standpoint of extending the data, doing a study to evaluate how bowel function is affected by epidurals, because when I talk to my anesthesia colleagues about whether there is a chance that patients really get an ileus from an epidural, they all say that that is not possible. For me, at least, this has been fairly convincing, and for the time being I have not considered a randomized trial. 